\title{
Remotely Powered Telemetry System with Dynamic Power-Adaptation for Freely Moving Animals
}

\author{
Enver G. Kilinc $^{\star}$, Franco Maloberti ${ }^{\diamond}$, and Catherine Dehollain ${ }^{\star}$ \\ * RF-IC Group, Ecole Polytechnique Fédérale de Lausanne, Lausanne, Switzerland \\ $\diamond$ Integrated Microsystem Laboratory, Universitá degli Studi di Pavia, Pavia, Italy \\ Email: enver.kilinc@epfl.ch, franco.maloberti@unipv.it, catherine.dehollain@epfl.ch
}

\begin{abstract}
A complete implantable system for monitoring vital parameters via bio-sensors inside freely moving laboratory animals and its experimental verification are presented. The system harvests $2 \mathrm{~mW}$ power wirelessly and the power is transmitted at $10 \mathrm{MHz}$ by an array of coils placed under the living space of the animal. Dynamic power-adaptation is used to transfer sufficient power to implanted module by adjusting the base module power with respect to animal position. Results on the micro-modules incorporating custom 0.18 um CMOS circuits verify a $80 \%$ power efficiency at $2 \mathrm{~mW}$ load and $60 \mathrm{~dB}$ of ripple suppression, as required to supply the implanted bio-sensors.
\end{abstract}

\section{INTRODUCTION}

The laboratory animals are essential in developing new treatments and research applications. Especially, the transgenic mice are used in research due to their smaller size, ability to grow up quickly compared to the rats and capability of modifying of their DNA sequence such that it allows us to have high resemblance to human genome [1]. On the electronic processing and communication side, there are many challenges that must be resolved concurrently. The electronic system developed for mice must be extremely small and light-weight in order to be implantable inside a mouse. Consequently, the electronic system should be batteryless. Although the battery can be a solution for extending the duration of measurements, it must be changed at the end of its lifetime and occupies large space compared to electronic circuits.

The implantable bio-sensor systems are able to detect different kind of endogenous compounds and/or drugs in the metabolism. The targeted remotely powered bio-sensor system is consisted of several electrochemical sensors based on cytochrome $\mathrm{P} 450$ isoforms, and on-chip $\mathrm{pH}$ and temperature sensors. Therefore, this bio-electronic-sensor system can detect the levels of different drugs according to different $\mathrm{pH}$ and temperature levels of the metabolism [2]. Fig. 1 illustrates the implantable bio-sensor system for a laboratory mouse. The system is composed of three layers. First layer is power coil which converts the magnetic field to induced current. Second layer includes the electronic circuits for the sensor system. The sensors are placed on the top layer for ensuring the contact with the metabolism.

Previous studies show that the measurement data should be obtained in the proper manner, because the condition of the subject animal changes the result of the measurement [3]. Therefore, the mouse should be in conscious and able to freely move in a defined space in order to give rise to correct data

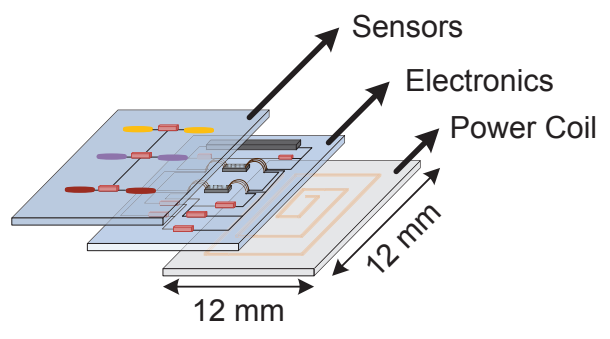

Fig. 1. Implantable sensor system for laboratory mouse

to be measured. In addition, a continuous remote powering system must be guaranteed for real-time long-term monitoring when the mouse moves freely in the available space.

It is possible to harvest the power wirelessly by using many different methods such as electromagnetic wave and magnetic field. The optimal choice is using magnetic field as it is more effective for short distance between the transmitter and the receiver modules [4]. The system described here includes the power and data transmission and the $2 \mathrm{~mW}$ supply generator for an implanted bio-sensor. The use of micro-modules with dedicated chips make the system flexible and usable for other kind of implanted sensors.

\section{Remote Powering Link For Implantable SEnsor SYSTEM}

The remote powering link is the most crucial part in the complete system due to the low coupling between the coils. The overall power efficiency is dominated by the remote powering link efficiency. Therefore the coils should be optimized for maximizing the power transfer efficiency [5]. However, the size of the optimized powering coil is insufficient to cover the bottom of the mouse living space. Therefore, an array of powering coils is placed under that space as shown in Fig. 2. However, the power consumption of the external unit augments when increasing the number of the coils. In order to solve this problem, an intelligent powering system is proposed in [6]. This system turns on the most appropriate powering coil among the array of coils and turns off the redundant coils according to the position of the mouse. Although, the proposed solution decreases the overall power consumption, it can be improved by creating a closed feedback loop in order to reduce more power consumption of the overall system. This paper 


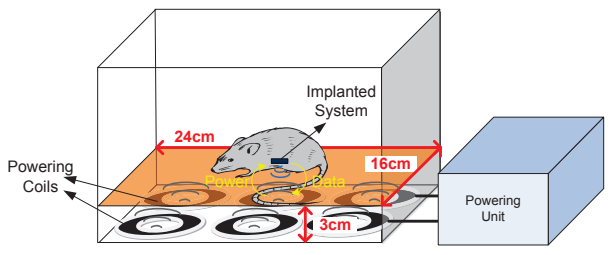

Fig. 2. Scenario for magnetically-coupled remote powering system for mice

presents a dynamic power-adaptation loop which optimizes the power level of the wireless transmission with respect to the position of the implanted coil on the selected powering coil.

\section{Electronic Circuits for Implantable SENSOR SYSTEM}

\section{A. External Module}

The power is transferred from the external module to internal module and data is transmitted from the internal module to the external module by using two different channels, because data requires higher frequency due to larger bandwidth. In addition, the bit error rate is higher when the mouse moves freely if the same channel is used with remote powering. Fig. 3 shows the block diagram of the remotely powered implantable sensor system. The external module includes powering and communication circuits. A power amplifier enhances the transmitted signal to augment the range of operation. For this function we use a class-E type power amplifier (PA). It allows powering coils with high power efficiency. A receiver (RX) detect the signal transmitted by the implanted module.

The mouse moves freely during remote powering. However, the power transmission is maximized when the external and the internal powering coils are placed concentrically. Consequently, the received power by the implanted unit is not always constant and changes according to the position of the mouse with respect to the external powering coil. In order to guarantee the minimum disturbance in the implanted unit, it is necessary to transfer the minimum power level that ensure operation. The power amplifier needs to transmit large amount of power when the mouse is at the edge of the external powering coil. Also, the internal module requires small amount of power when the mouse is at the center of the external coil. For these reasons, the transmitted power level should be adjusted to operate efficiently. A supply controller circuit performs this function by changing the supply of the power amplifier. The supply controller takes information from RX by using the output voltage of the implanted rectifier. If the voltage level is insufficient, the supply controller increases the supply voltage of PA until the voltage level of the internal module is adequate. On the contrary, when the voltage level is high, the supply controller reduces the supply of PA. Additionally, during the start-up condition, the implanted system has lack of power in order to turn on the operations. Therefore, TX cannot send any power data to RX. In such a case, the PA supply voltage increases slowly until the implanted unit turns on and the

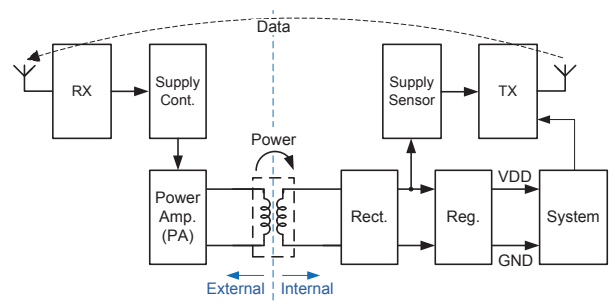

Fig. 3. Block diagram of remotely powered implantable sensor system

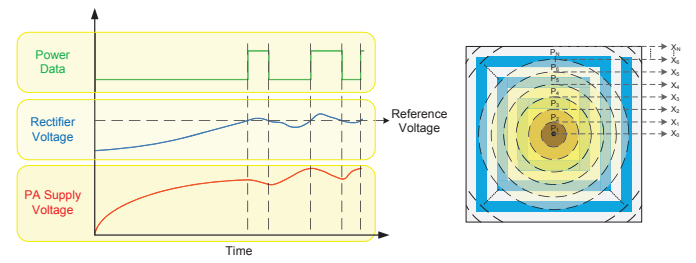

Fig. 4. Dynamic power-adaptation technique and iso-power curves

power data is received by RX. Fig. 4 illustrates the dynamic power-adaptation technique. In addition, this technique allows to measure the position of the mouse by tracking the supply voltage of the PA. The base module power levels are quantized in iso-power curves as shown in Fig. 4 and each iso-power curve represents a certain distance from the center of the coil by assuming the mice spend most of the time on their four feet [1]. Therefore, the position of the mouse can be measured by tracking the supply voltage of the PA. Furthermore, the activity of the mouse, which affects the vital signs of the body during the measurement, is recorded to examine the measured data in proper manner.

\section{B. Internal Module}

The rectifier and the regulator blocks generate the supply voltage of the whole system. This system uses a full-wave passive rectifier for ensuring low power consumption requirement. Fig. 5 shows the schematic of the full-wave rectifier circuit. In positive cycle of the AC signal, $M_{1}$ and $M_{3}$ transistors turn on. While, $M_{2}$ and $M_{4}$ transistors turn on in the negative cycle of the AC signal. During the start-up period, only $M_{1}$ and $M_{2}$ work as diode. However, since the voltage drop between source and drain $\left(V_{S D}\right)$ of $M_{1}$ and $M_{2}$ is quite large the efficiency of the rectifier can be affected. In order to moderate the problem, $V_{S D}$ voltage of $M_{3}$ and $M_{4}$ transistors is reduced by applying charge-storing technique [7], [8]. Initially, $V_{\text {out }}$ voltage is increased to $V_{a c+}-\left|V_{T P_{M 1}}\right|$ by $M_{1}$ transistor when $V_{a c+}$ is larger than threshold voltage of $M_{1}$ as shown in Fig. 5. As $V_{\text {out }}$ voltage increases, $M_{5}$ is turned on and charges $C_{1}$ capacitance at each positive cycle of $V_{a c+}$. Consequently, $V_{C 1}$ which is the gate voltage of $M_{3}$ transistor is reached to:

$$
V_{C 1}=V_{\text {out }}-\left|V_{T P_{M 5}}\right|=V_{a c+}-\left|V_{T P_{M 1}}\right|-\left|V_{T P_{M 5}}\right|
$$

Since the voltage between the source and the gate of $M_{3}$ $\left(V_{S G_{M 3}}\right)$ is $\left|V_{T P_{M 1}}\right|+\left|V_{T P_{M 5}}\right|>\left|V_{T P_{M 3}}\right|, M_{3}$ will be turned on and charge the output capacitance. Finally, the $V_{S D}$ voltage 


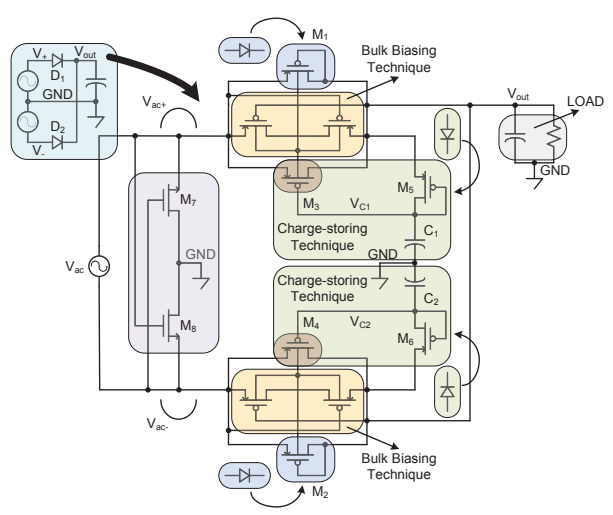

Fig. 5. Proposed full-wave rectifier circuit

of $M_{3}$ is decreased compared to diode connected PMOS transistor by applying charge-storing technique and shown as in [7]:

$$
V_{\text {out }}=V_{a c+}-\left(\left|V_{T P_{M 3}}\right|-\left|V_{T P_{M 5}}\right|\right)
$$

In addition, two PMOS transistors are used to bias the bulks of the $M_{3}$ and $M_{4}$ transistors as presented in Fig. 5 [9], [10]. Also, $M_{7}$ and $M_{8}$ NMOS transistors generates ground for the whole system.

Since full-wave rectifier works at positive and also negative phases of the input signal. The output of the rectifier is twice the one of the remote powering frequency. A voltage regulator suppresses the ripples and generates a clean supply voltage. Another request is that the supply voltage of the whole system must be always stable for all load conditions. The use of a linear low drop-out voltage regulator (LDO) ensures the voltage quality as required by the implantable system with a limited volume used. Indeed the LDO does not need extra inductors and/or capacitors but only active devices. In addition, the use of a linear regulator limits noise and complexity. Fig. 6 shows the linear low drop-out voltage regulator. It is a conventional scheme; however, since the ripples on the rectifier output voltage are at high frequency, the speed of the regulator must be also high. In order to minimize the power, a single stage OTA drives the pass transistor.

The power level of the implanted unit is monitored to ensure that while the laboratory mouse moves a sufficient power is transmitted to internal module. The supply sensor outlined in Fig. 3 is formed of a voltage divider and a comparator. Thus, the output voltage of the rectifier is tracked by the comparator. When the rectifier voltage level is under the reference voltage, the comparator gives "0" as power feedback data. The power feedback data turns "1" when the rectifier output increases above the reference voltage as shown in Fig. 4. The power feedback data is transmitted by the transmitter (TX) from internal module to the $\mathrm{RX}$ in the external module and the transmitted power level is adapted according to these feedback data.

The full-wave rectifier, the LDO voltage regulator, and the voltage comparator are fabricated in $0.18 \mu \mathrm{m}$ standard CMOS process. The $3.3 \mathrm{~V}$ thick oxide transistors are used in order to

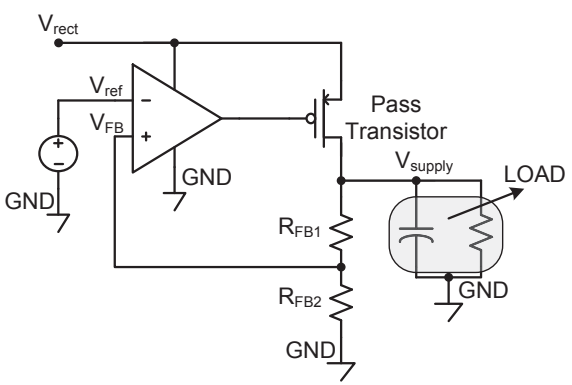

Fig. 6. Linear low drop-out voltage regulator

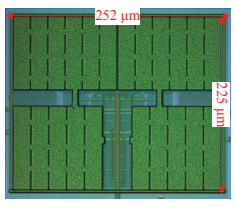

a) Full-wave Rectifier

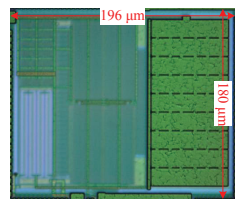

b) LDO Voltage Regulator

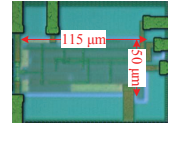

c) Voltage Comparator
Fig. 7. Micrograph of the fabricated (a) full-wave rectifier, (b) LDO voltage regulator, and (c) voltage comparator

increase the validity and the longevity of the circuits. Fig. 7 shows the micrograph of the fabricated circuits.

\section{Simulation \& Measurement Results}

The effectiveness of the powering link determined by the external and the internal modules has been verified by simulations and experimental measurements. Fig. 8 shows the experimental testbench. The external module comprises the RX, the supply controller, the PA and the external powering coil. The implanted powering coil, the full-wave rectifier, the LDO voltage regulator, the supply sensor, and the TX make the internal module. The implanted coil is placed $3 \mathrm{~cm}$ away from the external powering coil.

In order to have a term of comparison the entire system has been described at the behavioral level and simulated. Fig. 9 compares simulated and measured power efficiency of the proposed full-wave rectifier for different load conditions at $10 \mathrm{MHz}$ frequency. As shown in the graphic, the efficiency increases up to a certain load condition and decreases after a given point. It is due to the fact that the rectifier uses some current to charge the capacitances $\left(C_{1}\right.$ and $\left.C_{2}\right)$ as shown in Fig. 5 at each cycle of the operation frequency. In low power

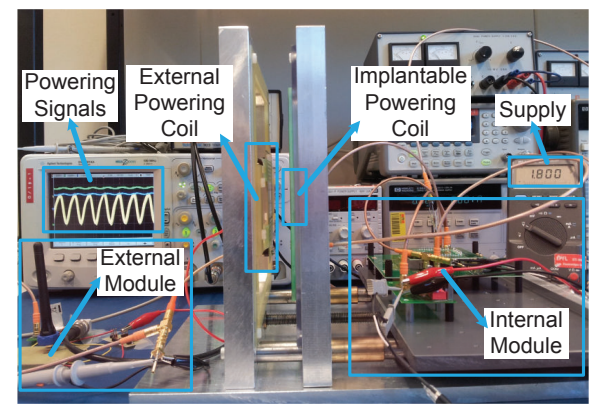

Fig. 8. Experimental testbench for remotely powered telemetry system 


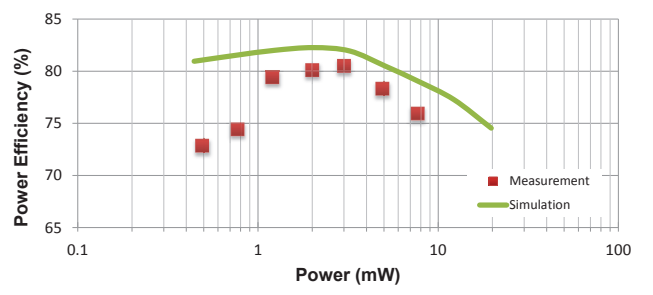

Fig. 9. Simulated and measured power efficiency of proposed rectifier

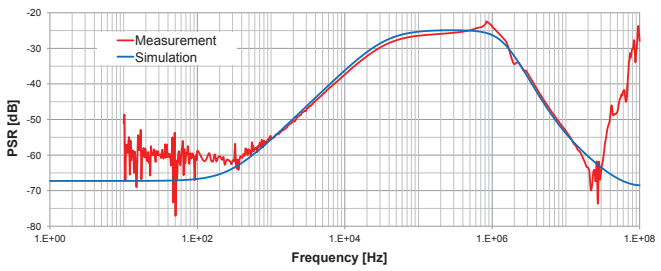

Fig. 10. Simulated and measured power supply rejection (PSR) response

consumption cases, the current of the rectifier circuit is low and some of the current is used by charge-storing technique inside the rectifier. Therefore, the efficiency is lower. For the high power consumption, the current of the rectifier is high and it creates a higher voltage drop over rectifier circuit which causes higher power consumption. Therefore, the rectifier is optimized to reach an optimum load condition which is similar to the power consumption of the implanted sensor system.

The LDO voltage regulator is situated after the rectifier to create a stable and clean $1.8 \mathrm{~V}$ voltage supply for the sensor system. In order to measure the PSR of the proposed LDO, the testbench which is described in [11] is used. Fig. 10 shows the simulated and measured power supply rejection (PSR) response of the circuit at $1 \mathrm{~mA}$ load condition from $10 \mathrm{~Hz}$ to $100 \mathrm{MHz}$. The simulation and measurement results fit well with exception of very low and very high frequencies. These differences are probably caused by the limitation of the measurement device.

The dynamic power-adaptation technique has been verified with the experimental setup of Fig. 8. Since the drop-out voltage of the regulator is $0.3 \mathrm{~V}$ and some safety margin is needed in order to guarantee continuation of the operation, the reference voltage is chosen as $2.4 \mathrm{~V}$ and the output of the rectifier voltage is compared with $2.4 \mathrm{~V}$ by the comparator. The result of the comparison, transmitted to the external module, controls the supply of the PA and hence changes the magnetic field strength. Fig. 11 shows the dynamic power-adaptation for the implantable sensor systems. As expected, the rectifier output voltage swings around $2.4 \mathrm{~V}$ : in the measured system it ranges between $2.31 \mathrm{~V}$ and $2.46 \mathrm{~V}$.

\section{CONCLUSION}

In this paper, a remotely powered telemetry system with dynamic power-adaptation for freely moving animals is presented. The power is transfered remotely over an optimized inductive link by using a class-E power amplifier at 10 $\mathrm{MHz}$ frequency. Furthermore, the transfered power is adjusted

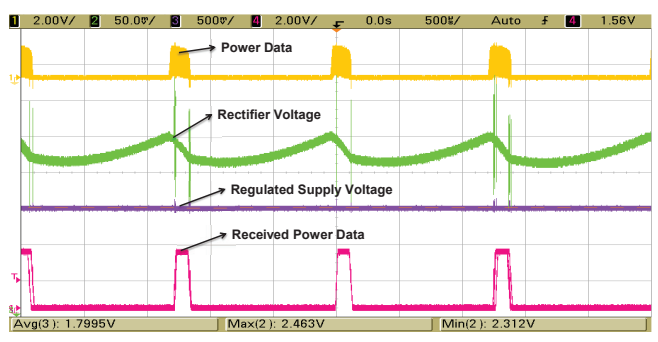

Fig. 11. Dynamic power-adaptation for implantable sensor systems

according to the power feedback data sent by the implanted system. This adjustment allows not only always optimized power transmission but also reduction in animal exposure to electromagnetic field which is also limited by the regulations. The transfered signal is rectified by a full-wave charge-stored passive rectifier with $80 \%$ power efficiency for $2 \mathrm{~mW}$ load condition. The ripples are suppressed more than $60 \mathrm{~dB}$ at the desired frequency by a high-speed LDO voltage regulator. Experimental results show that the rectifier voltage remains around $2.4 \mathrm{~V}$ and changes between $2.31 \mathrm{~V}$ and $2.46 \mathrm{~V}$ for transmitting sufficient power for implanted system. Therefore, experimental measurements confirmed by simulation results indicate the suitability of the system for powering and transmitting low-speed data from the implanted battery-less micromodule to the external module.

\section{ACKNOWLEDGMENT}

The authors thank to A.C. Moya, F. Mazzilli, G. Yilmaz, K.M. Silay, and O. Atasoy for their valuable feedback and help. This project is supported by Swiss National Funding (SNF) through Sinergia Initiative.

\section{REFERENCES}

[1] J. N. Crawley, What's Wrong With My Mouse: Behavioral Phenotyping of Transgenic and Knockout Mice, 2nd ed. Hoboken,NJ:Wiley, 2007.

[2] S. Carrara, et. al., "Multiplexing $\mathrm{pH}$ and temperature in a molecular biosensor," in IEEE Biomedical Circuits and Systems Conference, pp. 146-149, 2010.

[3] Q. Wang, H.R. Brunner, M. Burnier, "Determination of cardiac contractility in awake unsedated mice with a fluid-filled catheter," in Am. J. Physiol. Heart Circ. Physiol., vol. 286, no. 2, pp. H806-814, 2004.

[4] D.C. Yates, A.S. Holmes, A.J. Burdett, "Optimal transmission frequency for ultralow-power short-range radio links," in IEEE Trans. Circuits Syst. I, vol. 51, no. 7, pp. 1405-1413, 2004.

[5] E.G. Kilinc, C. Dehollain, F. Maloberti, "Design and optimization of inductive power transmission for implantable sensor system," in Proc. SM2ACD'10, pp. 1-5, 2010.

[6] E.G. Kilinc, et. al., "Intelligent cage for remotely powered freely moving animal telemetry systems," in Proc. IEEE ISCAS'12, pp. 2207-2210, 2012.

[7] T.T. Le, et. al., "Piezoelectric micro-power generation interface circuits," in IEEE J. Solid-State Circuits, vol. 41, no. 6, pp. 1411-1420, 2006.

[8] S. Hashemi, M. Sawan, Y. Savaria, "Fully-integrated low-voltage highefficiency CMOS rectifier for wirelessly powered devices," in Proc. Circuits Syst. TAISA Conf., pp. 1-4, 2009.

[9] P. Favrat, P. Deval, M.J. Declercq, "A high-efficiency CMOS voltage doubler," in IEEE J. Solid-State Circuits, vol. 33, no. 3, pp. 410-416, 1998.

[10] M. Ghovanloo, K. Najafi, "Fully integrated wide-band high-current rectifiers for wireless biomedical implants," in IEEE J. Solid-State Circuits, vol. 39, no. 11, pp. 1976-1984, 2004.

[11] Texas Instruments Incorporated, USA. Ldo psrr measurement simplified: Slaa414, 2009. 